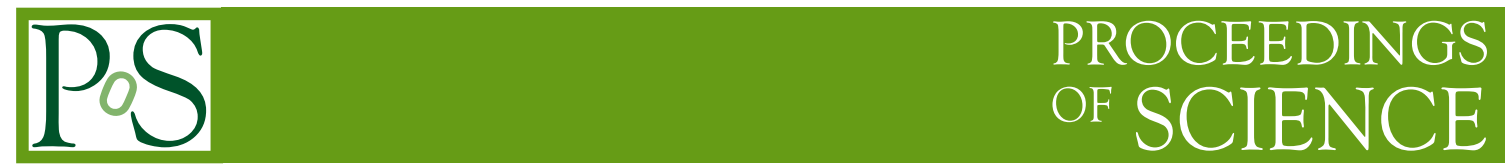

\title{
Proton structure and PDFs at HERA
}

\author{
Vladimir Chekelian ${ }^{* \dagger}$ \\ MPI for Physics, Munich \\ E-mail: shekeln@mail.desy.de
}

The neutral and charged current deep-inelastic $e p$ scattering cross sections are measured in the H1 and ZEUS experiments at HERA (1992-2007), with an electron beam energy of $27.6 \mathrm{GeV}$ and proton beam energies of $920,820,575$ and $460 \mathrm{GeV}$. In the second phase of the HERA operation (2003-2007) the lepton beam was longitudinally polarised. The data are used to determine the proton structure functions and are analysed in the QCD framework to obtain the parton distribution functions of the proton.

International Conference on the Structure and the Interactions of the Photon including the 20th International Workshop on Photon-Photon Collisions and the International Workshop on High Energy Photon Linear Colliders

20 - 24 May 2013

Paris, France

*Speaker.

${ }^{\dagger}$ on behalf of the H1 and ZEUS collaborations 


\section{Introduction}

Deep-inelastic scattering (DIS) data provide high precision tests of perturbative quantum chromodynamics (QCD), and have led to a detailed and comprehensive understanding of proton structure.

At the electron-proton collider HERA (1992-2007) electrons and positrons of $27.6 \mathrm{GeV}$ collided with protons of $920 \mathrm{GeV}$ ( $820 \mathrm{GeV}$ until 1997) corresponding to an ep centre-of-mass energy of $319 \mathrm{GeV}$. The operation of HERA proceeded in two phases, HERA I (1992-2000) with an unpolarised and HERA II (2003-2007) with a longitudinally polarised lepton beam. At the end of the HERA data taking, special runs with reduced proton beam energies of 460 and $575 \mathrm{GeV}$ were taken for dedicated $F_{L}$ measurement. The two $e p$ interaction regions were instrumented with the multi-purpose detectors of the $\mathrm{H} 1$ and ZEUS experiments.

The inclusive neutral (NC) and charged current (CC) ep DIS cross section data obtained by the $\mathrm{H} 1$ and ZEUS experiments are used to determine the proton structure functions and the partonic structure of the proton within the QCD framework. The HERAPDF sets of parton distribution functions (PDFs) are determined in the NLO and NNLO QCD fits of inclusive HERA data and also in fits including the charm and jet production data from HERA.

\section{Neutral and Charged Current Cross Sections}

The NC cross section for $e^{ \pm} p$ scattering can be expressed in a reduced form in terms of generalised proton structure functions $\tilde{F}$ as

$$
\tilde{\sigma}_{N C}^{ \pm}\left(x, Q^{2}\right)=\frac{\mathrm{d}^{2} \sigma_{N C}^{e^{ \pm} p}}{\mathrm{~d} x \mathrm{~d} Q^{2}} \frac{x Q^{4}}{2 \pi \alpha^{2}} \frac{1}{Y_{+}}=\tilde{F}_{2}\left(x, Q^{2}\right) \mp \frac{Y_{-}}{Y_{+}} x \tilde{F}_{3}\left(x, Q^{2}\right)-\frac{y^{2}}{Y_{+}} \tilde{F}_{L}\left(x, Q^{2}\right),
$$

where $\alpha$ is the fine structure constant, $x$ is the Bjorken scaling variable, $Q^{2}$ is the negative fourmomentum-transfer squared, and $y$ characterises the inelasticity of the interaction with $Y_{ \pm}=1 \pm$ $\left(1-y^{2}\right)$.

The generalised structure functions $\tilde{F}_{2,3}$ may be written as linear combinations of the structure functions $F_{2}, F_{2,3}^{\gamma Z}$ and $F_{2,3}^{Z}$, which contain information on QCD parton dynamics as well as on the electroweak couplings of the quarks to the neutral vector bosons. The $F_{2,3}^{\gamma Z}$ and $F_{2,3}^{Z}$ contributions to $\tilde{F}_{2,3}$ depend on the longitudinal polarisation of the lepton beam. The electromagnetic structure function $F_{2}$ is associated with pure photon exchange, $F_{2,3}^{\gamma Z}$ correspond to photon- $Z$ interference, and $F_{2,3}^{Z}$ describe pure $Z$-exchange. In the quark-parton model (QPM) $\tilde{F}_{L} \equiv 0 . \tilde{F}_{L}$ contributes only at high $y$ and is expected in QCD to be negligible at large $x$ and $Q^{2}$.

The generalised proton structure functions depend on the charge of the lepton beam, on the lepton beam polarisation, defined as $P=\left(N_{R}-N_{L}\right) /\left(N_{R}+N_{L}\right)$, where $N_{R}\left(N_{L}\right)$ is the number of right (left) handed leptons in the beam, and on the electroweak parameters $M_{Z}$ and $\sin ^{2} \theta$ (or $M_{W}$ ):

$$
\begin{aligned}
& \tilde{F}_{2}^{ \pm}=F_{2}+k\left(-v_{e} \mp P a_{e}\right) F_{2}^{\gamma Z}+k^{2}\left(v_{e}^{2}+a_{e}^{2} \pm 2 P v_{e} a_{e}\right) F_{2}^{Z}, \\
& x \tilde{F}_{3}^{ \pm}=k\left(-a_{e} \mp P v_{e}\right) x F_{3}^{\gamma Z}+k^{2}\left(2 v_{e} a_{e} \pm P\left(v_{e}^{2}+a_{e}^{2}\right)\right) x F_{3}^{Z} .
\end{aligned}
$$

Here, $k\left(Q^{2}\right)=\frac{1}{4 \sin ^{2} \theta \cos ^{2} \theta} \frac{Q^{2}}{Q^{2}+M_{Z}^{2}}$ determines the relative amount of $Z$ to $\gamma$ exchange, $v_{e}=-1 / 2+$ $2 \sin ^{2} \theta$ and $a_{e}=-1 / 2$ are the vector and axial-vector couplings of the electron to the $Z$ boson, and $\theta$ is the electroweak mixing angle. 
In the QPM the structure functions $\tilde{F}_{2}$ and $x \tilde{F}_{3}$ are related to linear combinations of sums and differences of the quark and anti-quark momentum distributions $x q\left(x, Q^{2}\right)$ and $x \bar{q}\left(x, Q^{2}\right)$ :

$$
\begin{aligned}
{\left[F_{2}, F_{2}^{\gamma Z}, F_{2}^{Z}\right] } & =x \sum_{q}\left[e_{q}^{2}, 2 e_{q} v_{q}, v_{q}^{2}+a_{q}^{2}\right](q+\bar{q}), \\
{\left[x F_{3}^{\gamma Z}, x F_{3}^{Z}\right] } & =2 x \sum_{q}\left[e_{q} a_{q}, v_{q} a_{q}\right](q-\bar{q}) .
\end{aligned}
$$

Here, $v_{q}$ and $a_{q}$ are the vector and axial-vector couplings of the quarks to the $Z$ boson, respectively, and $e_{q}$ is the charge of the quark of flavour $q$.

The CC $e^{ \pm} p$ cross section can be expressed in the reduced form as

$$
\tilde{\sigma}_{\mathrm{CC}}^{ \pm}\left(x, Q^{2}\right) \equiv \frac{2 \pi x}{G_{F}^{2}}\left[\frac{M_{W}^{2}+Q^{2}}{M_{W}^{2}}\right]^{2} \frac{\mathrm{d}^{2} \sigma_{\mathrm{CC}}^{e^{ \pm}} p}{\mathrm{~d} x \mathrm{~d} Q^{2}}=\left(1 \pm P_{e}\right)\left(Y_{+} W_{2}^{ \pm} \mp Y_{-} x W_{3}^{ \pm}-y^{2} W_{L}^{ \pm}\right) / 2,
$$

where $G_{F}$ is the Fermi constant defined using the weak boson masses $M_{W}$ and $M_{Z}$. This equation shows that the cross section has a linear dependence on the longitudinal polarisation of the lepton beam $P_{e}$. In the QPM $W_{L}^{ \pm} \equiv 0$, and the structure functions $W_{2}^{ \pm}$and $x W_{3}^{ \pm}$are expressed as the flavour dependent sum and difference of the quark and anti-quark momentum distributions. Only positively charged quarks contribute to $W^{-}$mediated scattering, and only negatively charged quarks couple to the exchanged $W^{+}$boson:

$$
\begin{aligned}
W_{2}^{-} & =x(U+\bar{D}), \quad W_{2}^{+}=x(\bar{U}+D), \\
x W_{3}^{-} & =x(U-\bar{D}), \quad x W_{3}^{+}=x(D-\bar{U}) .
\end{aligned}
$$

Below the $b$ quark mass threshold, the $U, \bar{U}$ and $D, \bar{D}$ distributions represent the sums of up-type and down-type quark densities:

$$
U=u+c, \bar{U}=\bar{u}+\bar{c}, D=d+s, \bar{D}=\bar{d}+\bar{s} .
$$

\section{The inclusive $\mathrm{NC}$ and $\mathrm{CC}$ data at HERA}

The H1 and ZEUS measurements of inclusive DIS reactions [1, 2, 3, 4, 5, 6] extend over a wide range in $x$ and $Q^{2}$, from very low $x=6 \cdot 10^{-7}$ and $Q^{2}=0.045 \mathrm{GeV}^{2}$ to $x=0.65$ and $Q^{2}=50000 \mathrm{GeV}^{2}$ for the NC process, and for CC interactions the range in $x$ is $0.0013 \leq x \leq 0.65$ and in $Q^{2}$ from $300 \leq Q^{2} \leq 30000 \mathrm{GeV}^{2}$. Each of the two experiments, $\mathrm{H} 1$ and ZEUS, collected a total integrated luminosity of $\approx 0.5 f b^{-1}$, about equally shared between positive and negative polarities and positive and negative longitudinal polarisations of the lepton beam.

The $Q^{2}$ dependence of the NC and CC cross sections at HERA is shown in Figure 1 (left). The NC cross sections exceed the CC cross sections at $Q^{2} \approx 300 \mathrm{GeV}^{2}$ by more than two orders of magnitude. The cross sections are of comparable size at $Q^{2} \approx M_{Z}^{2}, M_{W}^{2}$, illustrating the unified behaviour of the electromagnetic and the weak interactions at large scales in DIS.

The polarisation effects in $\mathrm{CC}$ are studied using the total $\mathrm{CC}$ cross sections defined in the region $Q^{2}>400 \mathrm{GeV}^{2}$ and $y<0.9$. The results for the polarised total $\mathrm{CC}$ cross sections measured at HERA II are shown in Figure 1 (right) together with the unpolarised total cross sections measurements from HERA I. The Standard Model predicts that, in the absence of right-handed charged 

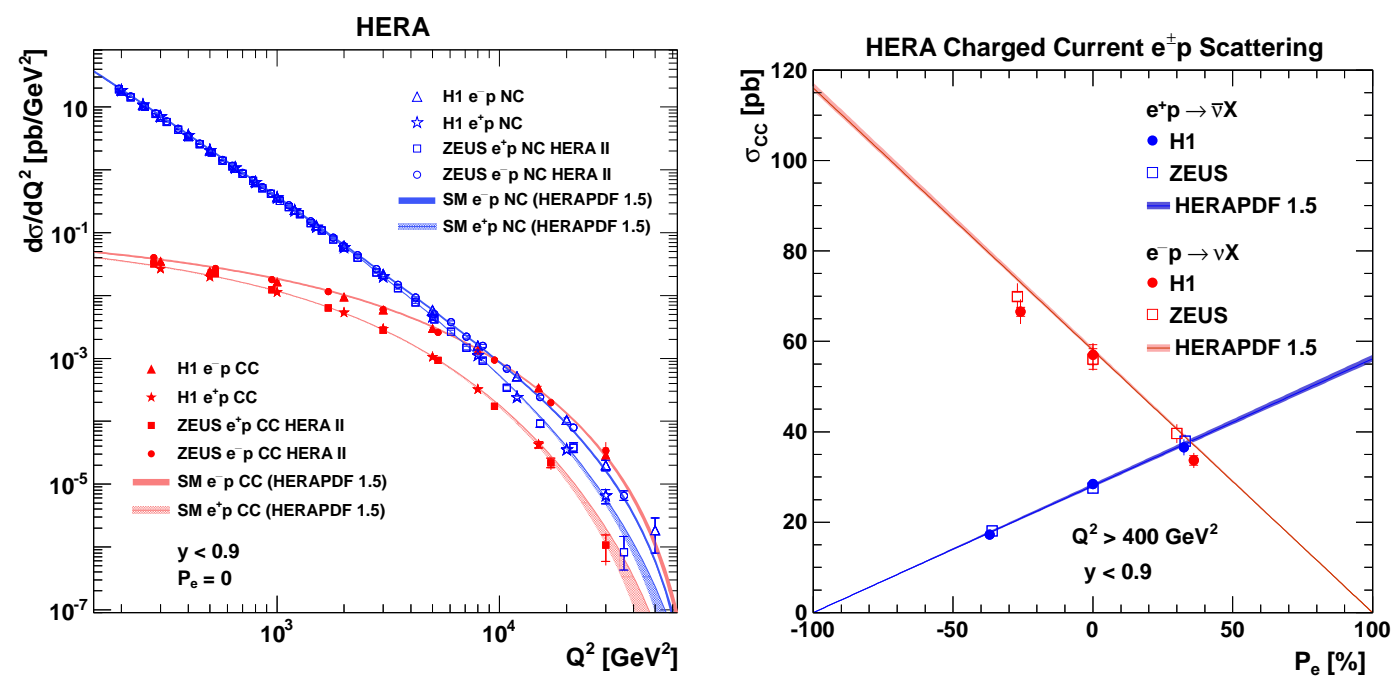

Figure 1: Left: The $Q^{2}$ dependence of the $e^{+} p$ and $e^{-} p$ NC and CC cross sections. Right: The dependence of the $e^{+} p$ and $e^{-} p$ CC cross sections on the lepton beam polarization $P_{e}$. The data are compared to the Standard Model prediction based on HERAPDF1.5 [15].

currents, the $e^{-} p\left(e^{+} p\right) \mathrm{CC}$ cross section is directly proportional to the fraction of right-handed electrons (left-handed positrons) in the beam. The measurements exhibit the expected linear dependence as a function of the polarisation $P_{e}$ and are consistent with the axiomatic absence of right-handed charged currents in the Standard Model.

The unpolarised double differential $\mathrm{NC}$ and $\mathrm{CC}$ cross sections in the reduced form as measured by H1 (HERA I+II) are shown in Figure 2. The $e^{+} p$ and $e^{-} p$ CC data are sensitive to different combinations of the light flavours in the proton. For example the $e^{+} p$ cross section shown in Figure 2 (right) is governed by the down-quark density of the proton. This allows for a flavour decomposition of the content of the proton in the QCD analysis of the HERA data.

\section{Structure functions $F_{2}, F_{2}^{\gamma Z}, x F_{3}^{\gamma Z}$ and $F_{L}$}

The NC cross section is dominated by the $F_{2}$ contribution, and the measured reduced NC cross section, shown in Figure 2 (left), is equivalent to the electromagnetic proton structure function $F_{2}$ in the bulk of the phase space. The dependence of the HERA II cross sections measurements on the longitudinal polarisation of the lepton beam is used to measure the parity violating structure function $F_{2}^{\gamma Z}$ for the first time [6]. For a given lepton charge the difference of the left $\left(P_{L}\right)$ and right $\left(P_{R}\right)$ polarised $\mathrm{NC}$ cross sections is sensitive to $F_{2}^{\gamma Z}$ as well as $x F_{3}^{\gamma Z}$ and $x F_{3}^{Z}$ as given by

$$
\frac{\tilde{\sigma}_{N C}^{ \pm}\left(P_{L}^{ \pm}\right)-\tilde{\sigma}_{N C}^{ \pm}\left(P_{R}^{ \pm}\right)}{P_{L}^{ \pm}-P_{R}^{ \pm}}=k\left[\mp a_{e} F_{2}^{\gamma Z}+\frac{Y_{-}}{Y_{+}} v_{e} x F_{3}^{\gamma Z}-\frac{Y_{-}}{Y_{+}} k\left(v_{e}^{2}+a_{e}^{2}\right) x F_{3}^{Z}\right] .
$$

By taking the difference of equation 4.1 for the $e^{+} p$ and $e^{-} p$ data, the terms proportional to $x F_{3}^{\gamma Z}$ and $x F_{3}^{Z}$ cancel, and $F_{2}^{\gamma Z}$ can be directly extracted using the measured $\mathrm{NC}$ cross sections [6]. Only a weak $Q^{2}$ dependence is expected, and therefore the measurements are transformed to a common 

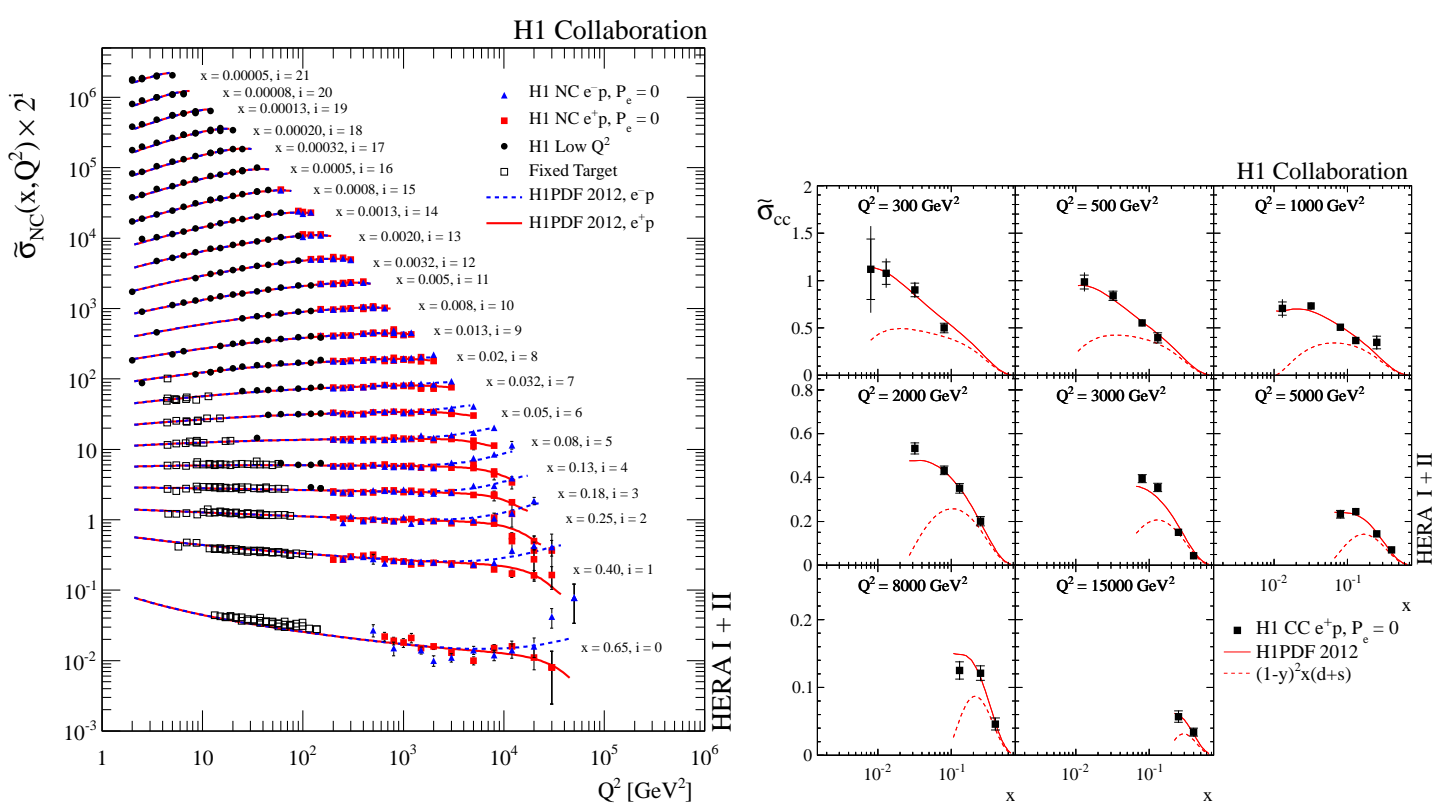

Figure 2: Left: The $e^{-} p$ and $e^{+} p$ NC reduced cross sections measured by H1 (solid symbols) using HERA I+II data are shown for various fixed values of $x$ as a function of $Q^{2}$. The fixed target data from BCDMS are also shown (open squares). Right: The $e^{+} p \mathrm{CC}$ reduced cross section measured by $\mathrm{H} 1$. The curves show the corresponding expectations using H1PDF 2012 [6]. In the figure on the right, the contribution $(1-y)^{2} x(d+s)$ is also shown.

$Q^{2}$ value of $1500 \mathrm{GeV}^{2}$ and are averaged in each $x$ bin. The result is displayed in Figure 3 (left) in comparison with the expectations from H1PDF 2012 [6].

The difference at high $Q^{2}$ between the unpolarised $e^{-} p$ and $e^{+} p$ NC cross section data in Figure 2 (left) is due to $x \tilde{F}_{3}$ entering Eq. 2.1 with opposite sign. This lepton beam charge dependence is used to measure the structure function $x \tilde{F}_{3}$, which is obtained from the cross section difference between electron and positron data:

$$
x \tilde{F}_{3}=\frac{Y_{+}}{2 Y_{-}}\left[\tilde{\sigma}_{N C}^{-}\left(x, Q^{2}\right)-\tilde{\sigma}_{N C}^{+}\left(x, Q^{2}\right)\right] .
$$

The dominant contribution to $x \tilde{F}_{3}$ arises from the $\gamma Z$ interference, which allows $x F_{3}^{\gamma Z}$ to be extracted according to $x F_{3}^{\gamma Z} \simeq-x \tilde{F}_{3} / k a_{e}$ by neglecting the pure $Z$-exchange contribution, which is suppressed by the small vector coupling $v_{e}$. The structure function $x F_{3}^{\gamma Z}$ is non-singlet and has little dependence on $Q^{2}$. The measured $x F_{3}^{\gamma Z}$ at different $x$ values can thus be averaged taking into account the small $Q^{2}$ dependence. The averaged $x F_{3}^{\gamma Z}$ determined as a function of $x$ for a $Q^{2}$ value of $1500 \mathrm{GeV}^{2}$ is shown in Figure 3 (right). The $x F_{3}^{\gamma Z}$ measurement is directly sensitive to the valence quark distributions and is well described by H1PDF 2012 [6].

The longitudinal structure function $F_{L}$ is obtained at HERA $[7,8,9,10]$ in a model independent way using measurements at fixed $x$ and $Q^{2}$ but different $y$. This is achieved using running periods with reduced $(460 \mathrm{GeV}$ and $575 \mathrm{GeV})$ and nominal $(920 \mathrm{GeV})$ proton beam energies. According to Eq. (2.1), the $F_{L}\left(x, Q^{2}\right)$ contribution to the reduced cross section is proportional to 

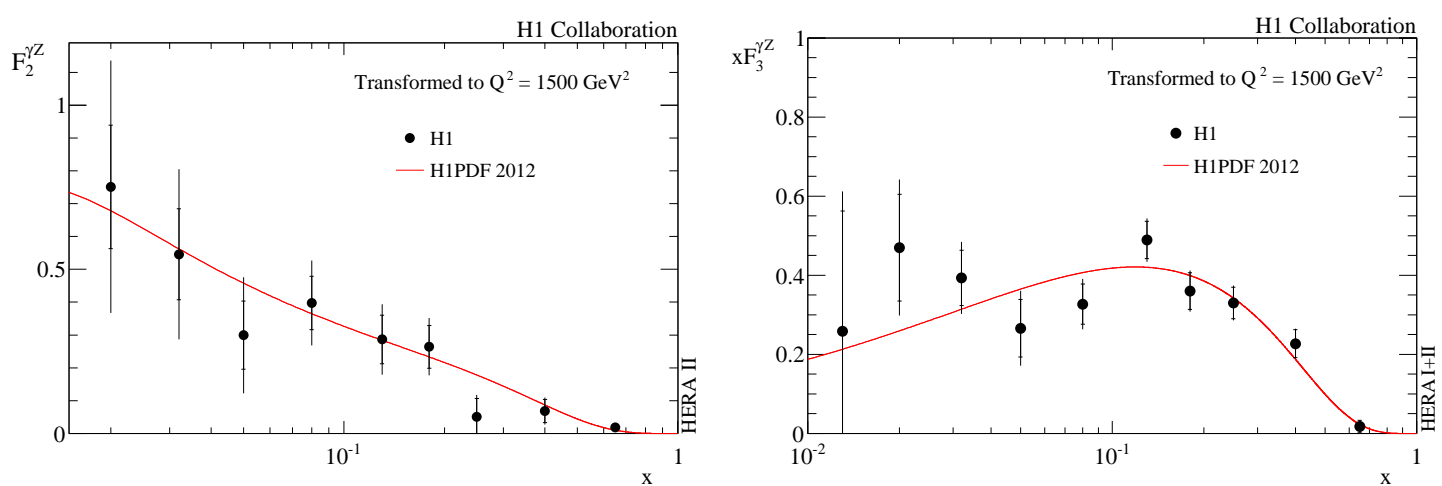

Figure 3: The structure function $F_{2}^{\gamma Z}$ (left) and the structure function $x F_{3}^{\gamma Z}$ (right) as a function of $x$ at $Q^{2}=1500 \mathrm{GeV}^{2}$ compared to predictions based on H1PDF 2012 [6].

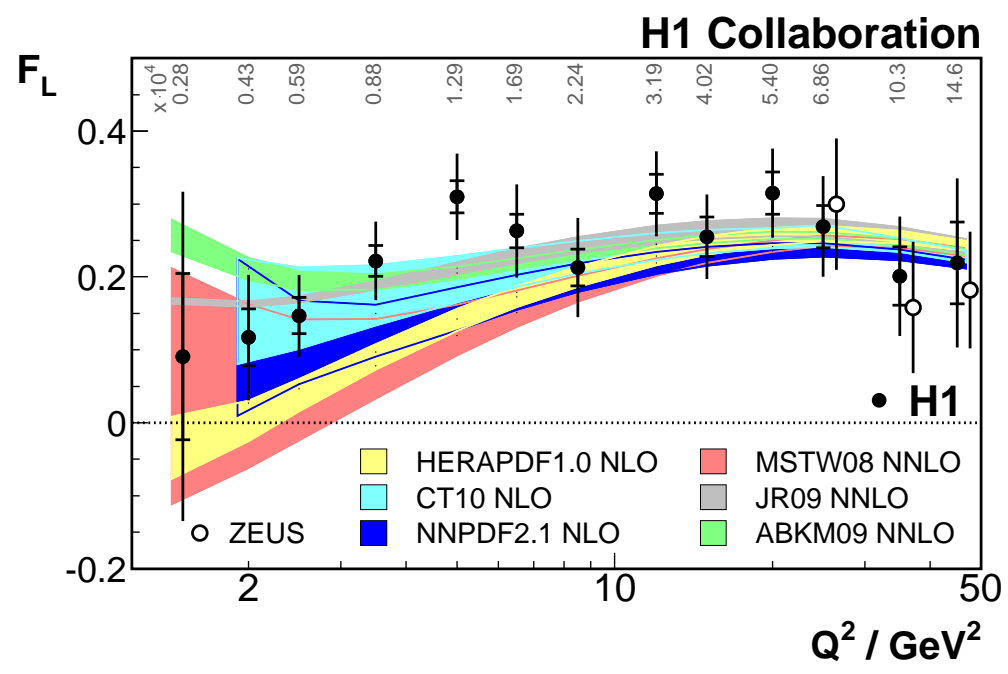

Figure 4: The H1 and ZEUS measurements of $F_{L}\left(Q^{2}\right)$ averaged over $x$ at fixed values of $Q^{2}$. The resulting $x$ values of the averaged $F_{L}$ are given in the figure on top for each point in $Q^{2}$. The data are compared to NLO and NNLO predictions based on a selection of PDF sets as indicated.

$f(y)=y^{2} /\left[1+(1-y)^{2}\right]$. Therefore, the $F_{L}$ values can be determined as slopes of straight-line fits of the measured $\tilde{\sigma}_{N C}\left(x, Q^{2}, y\right)$ values as a function of the $y$-dependent factor $f(y)$. More sophisticated procedures to determine $F_{L}$ and $F_{2}$ are applied by H1 and ZEUS to take into account correlations of data points due to systematic uncertainties. The H1 and ZEUS measurements of $F_{L}\left(x, Q^{2}\right)[9,10]$ are averaged over $x$ at fixed $Q^{2}$, and the resulting $F_{L}\left(Q^{2}\right)$ is shown in Figure 4. The data are reasonably well reproduced by theoretical predictions at NLO and NNLO. The measurements of $F_{L}$ are used to determine the ratio $R=F_{L} /\left(F_{2}-F_{L}\right)$. For $Q^{2} \geq 3.5 \mathrm{GeV}^{2}$ the ratio $R$ measured by $\mathrm{H} 1$ shows constant behaviour with $R=0.26 \pm 0.05$ [9]. 

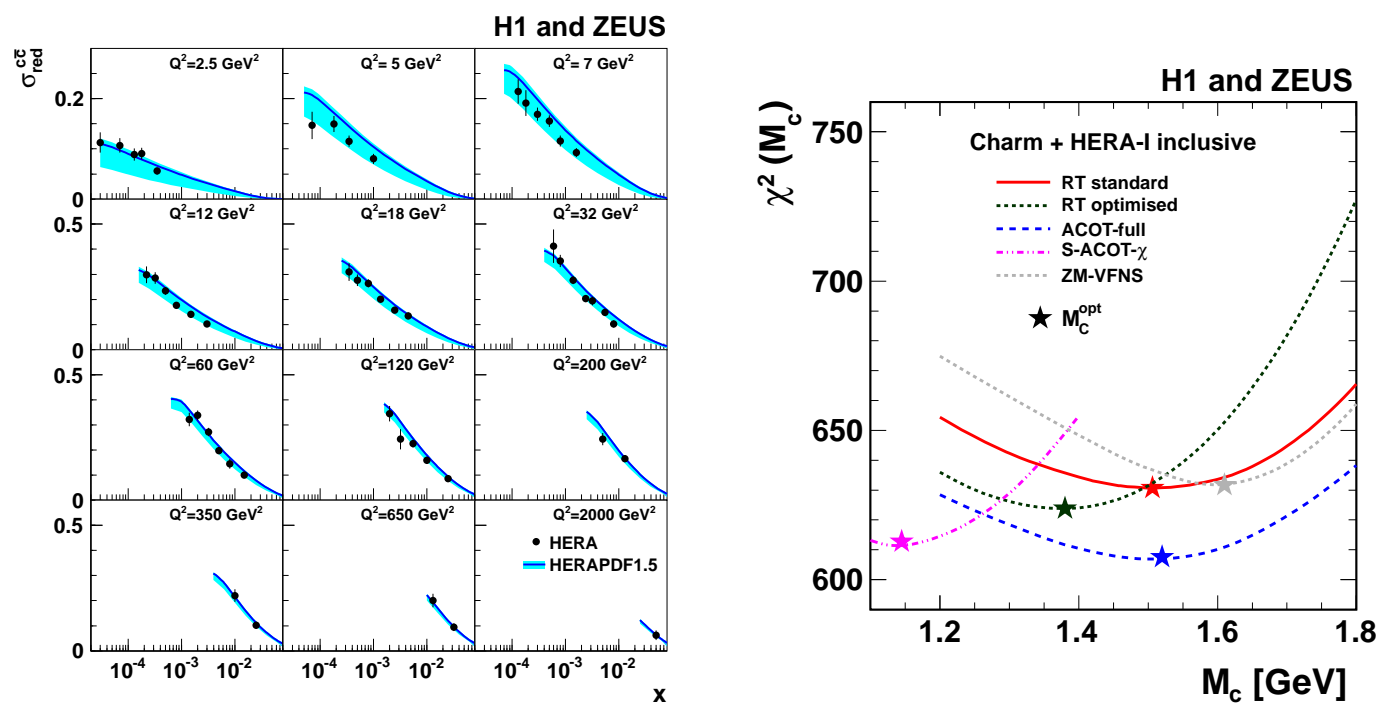

Figure 5: Left: Combined reduced charm cross sections at HERA as a function of $x$ for fixed values of $Q^{2}$. The data are compared to the NLO predictions based on HERAPDF1.5. Right: The values of $\chi^{2}\left(M_{c}\right)$ for the PDF fit to the combined HERA inclusive DIS and charm measurements. Different heavy flavour schemes are used in the fit and presented by lines with different styles. The values of the parameter $M_{c}^{\text {opt }}$ for each scheme are indicated by the stars.

\section{Combination of the $\mathrm{H} 1$ and ZEUS inclusive cross section data}

The H1 and ZEUS inclusive cross section data comprise many data sets which are obtained using different detector components and different running conditions. The HERA I inclusive NC and CC cross sections measured by $\mathrm{H} 1$ and ZEUS are combined in one simultaneous minimisation [1]. The resulting shifts of the correlated systematic uncertainties are propagated to both $\mathrm{NC}$ and $\mathrm{CC}$ data such that one coherent data set is obtained. Since H1 and ZEUS have employed different experimental techniques, different detectors and methods of kinematic reconstruction, the averaging leads to a significantly reduced correlated systematic uncertainty. The total uncertainty of the combined data set reaches the percent level for NC scattering in the best measured region. The averaging is extended [11] to include preliminary H1 and ZEUS measurements from the HERA II running period. The inclusion of the large HERA II data set leads to an improved uncertainty especially at high $Q^{2}$ and $x$. The final step to come is a combination of the entire HERA I+II inclusive data into one complete and coherent set of the inclusive $\mathrm{NC}$ and $\mathrm{CC}$ cross section data at HERA.

\section{Charm production cross section, $F_{2}^{c \bar{c}}$ and $m_{c}\left(m_{c}\right)$}

Measurements of open charm production in NC at HERA provide important input for tests of QCD and for the determination of the charm density in the proton. Similarly to the inclusive $\mathrm{NC}$ cross section, the reduced charm production cross section in $\mathrm{NC}$ can be written in terms of the 
structure functions $F_{2}^{c \bar{c}}\left(x, Q^{2}\right)$ and $F_{L}^{c \bar{c}}\left(x, Q^{2}\right)$ as

$$
\sigma_{\text {red }}^{c \bar{c}}\left(x, Q^{2}\right)=\frac{\mathrm{d}^{2} \sigma^{c \bar{c}}}{\mathrm{~d} x \mathrm{~d} Q^{2}} \frac{x Q^{4}}{2 \pi \alpha^{2}} \frac{1}{Y_{+}}=F_{2}^{c \bar{c}}\left(x, Q^{2}\right)-\frac{y^{2}}{Y_{+}} F_{L}^{c \bar{c}}\left(x, Q^{2}\right) .
$$

The contribution $F_{L}^{c \bar{c}}$, originating from the exchange of longitudinally polarised photons, is small in the kinematic range of the HERA measurements and reaches at most a few percent at high $y$.

Different techniques with different advantages and limitations have been used at HERA for the charm cross section measurements: the full reconstruction of $D$ or $D^{*}$ mesons, the long lifetime of heavy flavoured hadrons and their semi-leptonic decays. These data from H1 and ZEUS were accommodated into a common $Q^{2}-x$ grid and combined [12] into one data set in a similar manner as for the inclusive cross sections [1]. The resulting combined charm cross section is shown in Figure 5 (left). It is equivalent to the structure function $F_{2}^{c \bar{c}}$, which comprises a significant fraction, $20-25 \%$, of the proton structure function $F_{2}$. The combined cross section is in agreement with the prediction using HERAPDF1.5, albeit within a large uncertainty which is mostly related to variation of the input charm mass parameter $M_{c}$ in the QCD fit.

The combined charm data are used together with the combined inclusive DIS cross sections to perform a detailed QCD analysis [12] using different models of charm production in DIS. In the fixed-flavour-number scheme at NLO, using the $\overline{\mathrm{MS}}$ running mass definition, the running charm quark mass is determined to be $m_{c}\left(m_{c}\right)=1.26 \pm 0.05_{\text {exp }} \pm 0.03_{\text {mod }} \pm 0.02_{\text {param }} \pm 0.02_{\alpha_{s}} \mathrm{GeV}$. This value agrees well with the world average [13].

\section{HERAPDF sets of the parton distribution functions}

The inclusive HERA data are an indispensable input to the determination of modern parton distribution functions in the QCD PDF fits. H1 and ZEUS performed a series of PDF fits referred to as HERAPDF using HERA data as sole input to the fits. The HERAPDF QCD fitting framework is based on QCDNUM [14] for the NLO and NNLO QCD evolution. In the HERAPDF fits a variable-flavour-number scheme is used. The usage of data from HERA only, with well controlled systematic uncertainties, allows a $\Delta \chi^{2}=1$ criterion to be used for the determination of the experimental errors of the fit. The uncertainties of the PDFs comprise also variations of the model assumptions like $Q_{\min }^{2}, M_{c}, M_{b}$ and the uncertainty related to the variation of the form of the PDF parametrisations at the starting scale, $Q_{o}^{2}=1.9 \mathrm{GeV}^{2}$.

The QCD analysis of the combined HERA I data is used to obtain the set of parton distribution functions HERAPDF1.0 [1]. The preliminary combination of the HERA I and HERA II data is used in the HERAPDF1.5 fit [15]. Compared to the HERAPDF1.0, the HERA II data further constrain the high $x$ valence PDFs. The resulting PDFs have total uncertainties at the level of a few percent at low $x$ and are shown at $Q^{2}=10000 \mathrm{GeV}^{2}$, corresponding to the LHC kinematic domain, in Figure 6 (left). The HERAPDF1.5 set of PDFs is widely used these days for comparisons with the LHC data.

Using the combined charm cross sections together with the combined inclusive DIS data, an NLO QCD analysis is performed [12] based on different implementations of the variable-flavournumber-scheme. For each scheme, an optimal value of the charm mass parameter, $M_{c}^{\text {opt }}$, is determined as it is shown in Figure 5 (right). All schemes are found to describe the data well, as long 

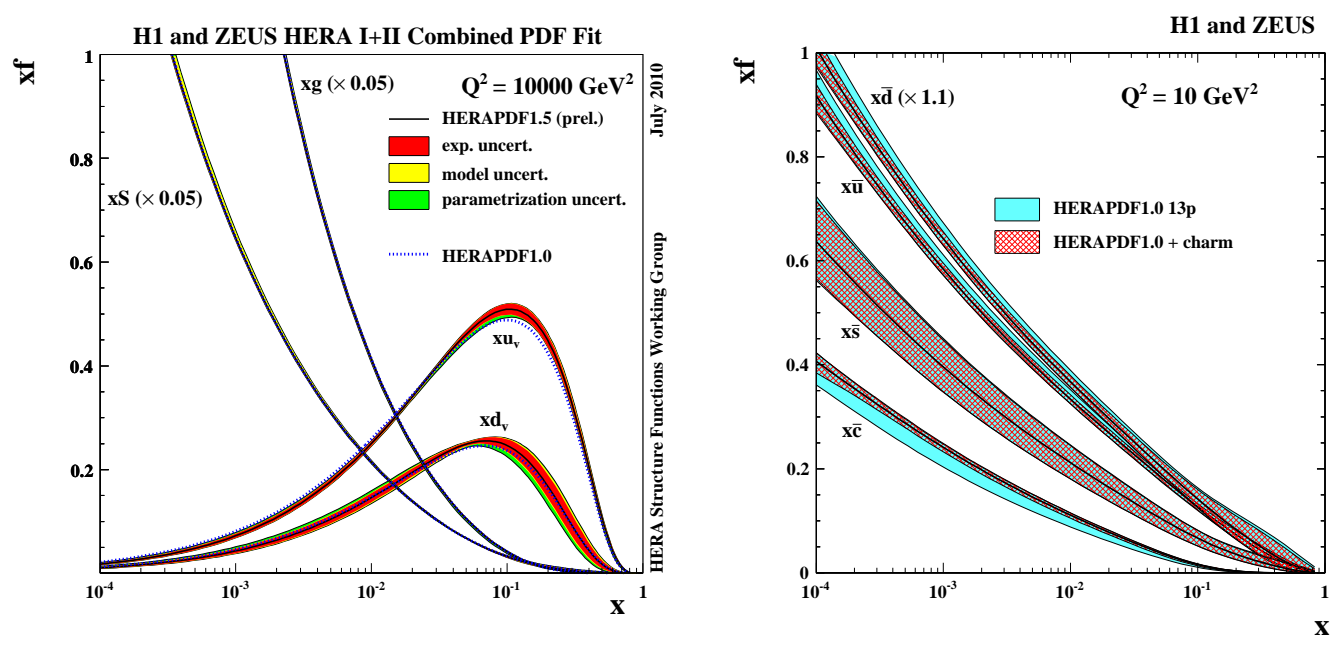

Figure 6: Left: Parton distribution functions of HERAPDF1.5 [15] at the evolved scale of $Q^{2}=10000 \mathrm{GeV}^{2}$. The gluon and sea distributions are scaled by a factor 0.05. Right: Sea anti-quarks obtained from the combined QCD analysis of the inclusive DIS and charm data as a function of $x$ at $Q^{2}=10 \mathrm{GeV}^{2}$. For comparison the results of the QCD analysis of the inclusive DIS data only are also shown (light shaded bands).

as the charm mass parameter is taken at the corresponding optimal value. Using optimal values of $M_{c}$, the predictions of $W^{ \pm}$and $Z$ production at the LHC become very stable with the spread due to different schemes within 2\%. As it is shown in Figure 6 (right) the use of the combined charm data reduces significantly the uncertainties for the sea quark contributions from charm, down and up quarks.

In the HERAPDF1.6 [16] fit the HERA jet data are fitted together with the preliminary combined HERA I+II inclusive data. The inclusion of the jet data in the fit makes simultaneous determination of the PDFs and the strong coupling $\alpha_{s}\left(M_{Z}\right)$ possible. Finally, the preliminary combined HERA I+II inclusive data, $F_{2}^{c c}$ and jet data from HERA are fitted together in the HERAPDF1.7 [17] fit.

\section{Summary}

The $\mathrm{H} 1$ and ZEUS experiments at HERA measured inclusive ep DIS cross sections over six orders of magnitude in $Q^{2}$ and $x$. Using these data the electromagnetic structure function $F_{2}$ is determined with an accuracy of about $1 \%$ in the best measured region. The proton structure functions $F_{2}^{\gamma Z}$ is measured for the first time. The structure functions $x F_{3}^{\gamma Z}$ and $F_{L}$ are measured as well. The H1 and ZEUS data are combined in a preliminary form and used to determine PDFs. The corresponding HERAPDF1.5 set of PDFs is widely used for comparisons with the LHC data. The H1 and ZEUS measurements of open charm production are combined and analysed in the fixedflavour-number scheme and in the general mass variable-flavour-number scheme within the QCD framework. 


\section{References}

[1] H1 and ZEUS Collab., F. D. Aaron et al., JHEP01, 109 (2010).

[2] ZEUS Collab., S. Chekanov et al., Eur. Phys. J., C61, 223 (2009).

[3] ZEUS Collab., S. Chekanov et al., Eur. Phys. J., C62, 625 (2009).

[4] ZEUS Collab., H. Abramowicz et al., Eur. Phys. J., C70, 945 (2010).

[5] ZEUS Collab., H. Abramowicz et al., Phys. Rev., D87, 052014 (2012).

[6] H1 Collab., F. D. Aaron et al., JHEP 09, 061 (2012).

[7] H1 Collab., F. D. Aaron et al., Phys. Lett., B665, 139 (2008).

[8] H1 Collab., H1prelim-08-042 (2008).

[9] H1 Collab., F. D. Aaron et al., Eur. Phys. J., C71, 1579 (2011).

[10] ZEUS Collab., S. Chekanov et al., Phys. Lett., B682, 8 (2009).

[11] H1 and ZEUS Collab., H1prelim-10-141, ZEUS-prel-10-017 (2010).

[12] H1 and ZEUS Collab., H. Abramowicz et al., Eur. Phys. J., C73, 2311 (2013).

[13] K. Nakamura et al. [Particle Data Group], J. Phys., G37, 075021 (2010).

[14] M. Botje, Comput. Phys. Commun., 182, 490 (2011).

[15] H1 and ZEUS Collab., H1prelim-10-142, ZEUS-prel-10-018 (2010).

[16] H1 and ZEUS Collab., H1prelim-11-034, ZEUS-prel-11-001 (2011).

[17] H1 and ZEUS Collab., H1prelim-11-143, ZEUS-prel-11-010 (2011). 